\title{
Knowledge to action for solving complex problems: insights from a review of nine international cases
}

\author{
B. L. Riley, PhD (1); K. L. Robinson, PhD (2); J. Gamble, MM (3); D. T. Finegood, PhD (4); D. Sheppard, MHSc (2); \\ T. L. Penney, MA (5); A. Best, PhD (6)
}

This article has been peer reviewed.

Tweet this article

\begin{abstract}
Introduction: Solving complex problems such as preventing chronic diseases introduces unique challenges for the creation and application of knowledge, or knowledge to action (KTA). KTA approaches that apply principles of systems thinking are thought to hold promise, but practical strategies for their application are not well understood. In this paper we report the results of a scan of systems approaches to KTA with a goal to identify how to optimize their implementation and impact.
\end{abstract}

Methods: A 5-person advisory group purposefully selected 9 initiatives to achieve diversity on issues addressed and organizational forms. Information on each case was gathered from documents and through telephone interviews with primary contacts within each organization. Following verification of case descriptions, an inductive analysis was conducted within and across cases.

Results: The cases revealed 5 guidelines for moving from conceiving KTA systems to implementing them: 1) establish and nurture relationships, 2) co-produce and curate knowledge, 3) create feedback loops, 4) frame as systems interventions rather than projects, and 5) consider variations across time and place.

Conclusion: Results from the environmental scan are a modest start to translating systems concepts for KTA into practice. Use of the strategies revealed in the scan may improve KTA for solving complex public health problems. The strategies themselves will benefit from the development of a science that aims to understand adaptation and ongoing learning from policy and practice interventions, strengthens enduring relationships, and fills system gaps in addition to evidence gaps. Systems approaches to KTA will also benefit from robust evaluations.

Keywords: intervention studies, public health, knowledge transfer, systems approach

\section{Introduction}

Public health problems such as preventing chronic diseases arise and persist as a result of multiple physiological, behavioural and environmental factors and their interactions. $^{1,2}$ Solutions to these complex pro- blems require new approaches to how knowledge is created and applied. ${ }^{3-5}$ In this paper we refer to these as "systems approaches for knowledge to action (KTA).”

Systems approaches to KTA build on linear approaches that emphasize effective packa-

\section{Key findings}

- Solving complex problems like preventing chronic diseases requires sharing and using knowledge of what works and how to support positive changes in communities.

- Our scan of 9 diverse examples of using knowledge for action found that there is no single formula or recipe for applying knowledge to specific problems; that solutions need to emerge and adapt over time based on feedback and evaluation; lasting, diverse relationships should be nimble and focus on sharing resources to inspire innovative thinking and solutions; and we need to build and use many types of knowledge together across sectors.

ging and dissemination of knowledge products and on relationship approaches that emphasize effective partnerships for developing and sharing knowledge. Systems approaches recognize that dissemination processes and relationships themselves are shaped, embedded and organized through structures that influence the types of interactions that occur among multiple stakeholders with unique worldviews, priorities, languages, means of communication and expectations. ${ }^{6}$ These stakeholders are tied together by a system (which in turn is shaped by culture, structures, priorities and capacities $^{7}$ ) that requires activation if its various parts are to be linked together. Consequently, a systems way of thinking is

\footnotetext{
Author references:

1. Propel Centre for Population Health Impact, University of Waterloo, Waterloo, Ontario, Canada

2. Public Health Agency of Canada, Ottawa, Ontario, Canada

3. Imprint Consulting, Hampton, New Brunswick, Canada

4. Simon Fraser University, Burnaby, British Columbia, Canada

5. Cambridge University, Cambridge, United Kingdom

6. InSource Research Group, Vancouver, British Columbia, Canada

Correspondence: Barbara Riley, Executive Director, Propel Centre for Population Health Impact, University of Waterloo, 200 University Ave. West, Waterloo, ON N2L 3G1;

Tel: 519-888-4567 ext. 37562; Fax: 519-746-8171; Email: briley@uwaterloo.ca
} 
needed to bring about that activation for the purposes of KTA. ${ }^{8,9}$

While the need for systems approaches to KTA is acknowledged, and concepts are gaining clarity, practical strategies to design and implement systems approaches to KTA are very limited..$^{8,10,11}$ The purpose of the environmental scan reported in this paper was to begin to show some practical strategies that may usefully guide the design, implementation and impact of KTA initiatives to solve complex problems.

Chronic diseases are a serious and urgent problem worldwide. In $2005,60 \%$ of total deaths worldwide were attributed to chronic diseases, and a $17 \%$ increase is projected from 2005 to $2015 .{ }^{12}$ Although it has been estimated that up to $50 \%$ of cancer, ${ }^{13} 90 \%$ of cardiovascular disease, ${ }^{14}$ and $91 \%$ of diabetes $^{15}$ are preventable, significant and scaled-up investments in population-level prevention interventions continue to be insufficient. Even modest reductions in chronic disease risk factors would save tens of thousands of person-years of life and hundreds of millions in direct health care costs worldwide each year. ${ }^{16,17}$

Chronic diseases are also complex problems. Their complexity arises from a dynamic interplay of factors that contribute to the development and persistence of chronic conditions. Factors span a wide range, including individual physiology and behaviour, institutional arrangements (e.g. health and social service organization interactions), and physical and social environments at local through societal levels (e.g. neighbourhood design, social cohesion, food supply and distribution). ${ }^{18}$

Complex problems such as chronic diseases do not respond to simple, independent, one-off solutions; they require deliberately coordinated sets of interventions and creative efforts at many jurisdictional levels (e.g. regional, provincial, national, international) ${ }^{4}$ and system levels (e.g. paradigm, goals, organizational structures). ${ }^{5,19}$ They require the engagement of actors and organizations involved with research, policy and practice and from health and non-health sectors. They also require tailoring policy and program interventions to diverse contexts and ongoing adaptation of interventions in dynamic environments, ${ }^{4}$ all with a goal to change health behaviours and the underlying conditions of risk in the case of chronic diseases.

Multi-faceted and dynamic solutions to complex problems introduce unique challenges for the creation and use of knowledge. $^{20-23}$ For example, it is now widely acknowledged that application of the concept of evidence-based medicine as originally conceived is not well-suited to public health interventions, ${ }^{24}$ making the direct application of research findings to various settings, time periods and populations problematic. In contrast, effective KTA approaches will be dynamic, multi-directional processes of engaging (often diverse) stakeholders to co-create, synthesize, share and use knowledge in order to inform decision-making and foster change in the contexts and based on needs of policy and program settings. ${ }^{25}$

Consequently, new approaches to evidence-informed public health are being explored and have led to many calls for the application of concepts and methods of systems science. ${ }^{8,23,26-30}$ Responses to these calls have resulted in a growing number of empirical studies that use systems modelling and network analysis techniques. These empirical studies have also contributed to the body of literature on the promise of applying systems thinking, variably defined, to knowledge development and use in population and public health. ${ }^{10,26-28,31,32}$ This emerging literature is relatively silent on the translation of concepts and principles of systems thinking into practical strategies for creating and using knowledge to solve complex problems.

Organizations such as the Public Health Agency of Canada and Propel Centre for Population Health Impact with panCanadian mandates to accelerate KTA for chronic disease prevention are attempting to fill this gap. The analysis and synthesis we describe in this paper are based on a scan of initiatives, all of which were addressing complex problems, included a focus on both creating and using knowledge, and applied principles of systems thinking in their KTA efforts.

\section{Methods}

A small advisory group (authors BR, KR, $\mathrm{DF}$, DS, $\mathrm{AB}$ ), representing public health research, policy and KTA organizations with a history of collaboration on projects related to KTA for chronic disease prevention, chose 3 main criteria to use in the search for organizations and their KTA initiatives: (1) intermediaries (as opposed to direct service delivery organizations) addressing a complex issue; (2) groups that are seeking to intervene at multiple levels in a system; and (3) groups that have used specific strategies for KTA informed by systems thinking.

Principles of systems thinking cover a wide range from many disciplines and schools of thought. For the scan, we integrated ideas from several systems thinkers ${ }^{3,34-36}$ and adapted them to a public health context. Through discussion and consensus among advisory group members, we developed 12 systems principles for solving complex public health problems and organized these principles into 3 overarching concepts: coherence, connectivity and continuous learning (see Table 1).

Using the 3 criteria for case selection, we identified an initial list of 30 KTA cases through a web-based search, a search of grey and published papers that explore complex or systems approaches to KTA, and by requesting nominations from people known to the advisory group. The initial set of cases were from Canada and other developed countries.

By reviewing publicly available documentation on the initial set of 30 potential cases, we chose 9 cases that best fitted the criteria and scope of the scan (see Table 2). We excluded cases from the analysis if they were direct service delivery organizations; intervened only at one level of a system (e.g. focussed only on individual behaviour change); or did not undertake KTA approaches (e.g. some organizations produced discussion papers on the concepts or theories of system approaches but did not undertake specific initiatives).

The 9 cases included in the scan were diverse, addressing a variety of areas and using different organizational forms. In 
TABLE 1

Principles of systems thinking for solving complex public health problems

\begin{tabular}{|c|c|c|}
\hline Coherence & Connectivity & Continuous learning \\
\hline Match capacity with complexity & Establish networks and teams & A reductionist paradigm is not that helpful \\
\hline Act locally, connect regionally, learn globally & Support individuals & Set functional goals \\
\hline Transformative leadership & Build authentic trust & Assess effectiveness \\
\hline Disruptive innovation & Distribute decision, action and authority & Linkage and exchange processes \\
\hline
\end{tabular}

some cases KTA was a core purpose (for example, ResearchImpact and Plexus) and in others it was an ancillary activity in support of other objectives (e.g. the National Treatment Strategy and Causeway). Some examples explicitly used a systems / complexity framing (e.g. Tamarack, Plexus and CEIPS), whereas others had features and characteristics consistent with a systems approach but did not use that language or framing directly (e.g. INSPIRE, ResearchImpact). Explicitly or implicitly, all of the organizations' KTA activities drew on principles of systems thinking.

A set of questions to guide documentation and analysis covered organizational vision, objectives, guiding principles, collaborators, activities, operational definitions, results and lessons learned. Initial data collection included analysis of publicly available documentation (reports/publications, website information) and a telephone interview with a primary contact within each organization who knew most about each case (e.g. the executive director or the project lead/coordinator). The primary contacts verified case descriptions and provided additional information, both directly and by recommending other reports that the advisory group had not previously reviewed.

We conducted an inductive review of the case documentation, informed by the system principles in Table 1, without being constrained by them. The principles were used as a preliminary analytic framework to examine how they were implemented in one or more cases. Themes related to practical strategies (e.g. people, processes, structures) were generated and coded in the case documentation. The analysis was completed by 2 researchers (authors JG, TP) and supplemented with reflections and analysis from the advisory group based on a review of documented examples of each theme. We then compared themes for similarities and differences, and identification of specific examples of themes were then completed across cases.

\section{Results}

Based on the scan analysis, we identified 5 guidelines on implementing systems approaches to KTA:

TABLE 2

Case organizations in the scan

\section{Organization}

Tamarack Institute - Vibrant Communities Canada (Toronto, Ontario, Canada)

http://tamarackcommunity.ca

Framework (Toronto, Ontario, Canada)

http://www.frameworkorg.org

Plexus Institute (Washington, DC, USA)

http://www.plexusinstitute.org

Centre of Excellence in Intervention and Prevention Science (CEIPS) (Victoria, Australia) http://ceips.org.au/

INSPIRE's EPISCentre (Pennsylvania State University, U.S.)

http://www.episcenter.psu.edu/

Researchlmpact-RéseaulmpactRecherche (RIR) (Canada)

http://www.researchimpact.ca/locaIRI/YorkU/

Social Innovation Generation (SiG) Causeway (Ontario)

http://www.sigeneration.ca

United Way Toronto (UWT) (Ontario, Canada)

http://www.unitedwaytoronto.com/

National Treatment Strategy- System Action Network (Canada)

http://www.nts-snt.ca/
Type of knowledge-to-action initiative

Example of an intermediary that puts learning, knowledge production and dissemination at the centre of a significant national poverty reduction initiative

Example of a small, creative organization that conducted a pilot with several organizations to explore how readily available technological tools could help the organizations gather and share evidence, practice and knowledge

Example of a capacity-building, action-research organization that was built explicitly around complexity and systems thinking

Example of a public health research centre

Example of a web-based evidence-gathering and dissemination tool as a centrepiece of KTA activities

Example of a KTA unit at a Canadian University that is also part of a multi-university collaboration aimed at supporting research use for policy and practice

Example of diverse organizations collaborating around a common theme

Example of an intermediary organization that has invested in a community of practice approach to mobilize knowledge in youth education

A national network and strategy for using a systems approach to address substance abuse treatment service gaps and client needs across government jurisdictions and various types of organizations 


\section{1) Establish and nurture relationships}

Building relationships was a central theme across cases, expressed in different organizational forms (e.g. networks, coalitions, advisory groups). The quality and depth of these connections beyond information sharing was apparent. Having advisors, partners and staff with experience in government, the private sector and the voluntary sector helps with gaining access to different individuals and groups, developing skills in navigating these relationships, and understanding the nuances of language and interpretation. For example, one of the core principles of the Vibrant Communities Initiative of the Tamarack Institute was establishing multisectoral collaborations in communities and providing supports to mobilize and sustain these collaborations. The range of perspectives from voluntary organizations, business leaders, government officials and people who had lived in poverty generated alternative options and built commitment to the strategies that were developed.

\section{2) Co-produce and curate knowledge}

Common across all cases was the understanding that KTA for complex problems requires shared interpretation, analysis and sense-making. Expert paradigms of knowledge creation and distribution are not helpful in the realm of the complex. If people who have ideas on how to improve practice are consistently disregarded or they have ideas they had not tried because of lack of time or space to implement them, the potential for change is limited. The metaphor of curating is useful in a few cases when thinking about systems approaches to KTA. Curating is about linking together elements that are similar and different. It is more than asking people what they want to know about; it anticipates what might be interesting and useful to policy and practice but may not be on the radar of relevant groups. For example, CEIPS embraced this approach by integrating research staff into committees of their local health authority to build relationships with practice and policy staff and bring their perspectives more directly into the research work.

\section{3) Create feedback loops}

The cases showed that KTA efforts for complex problems require context-specific results that convey what works (or does not), for whom, how and in what context. Multiple cases incorporate a feedback loop to help use the knowledge to inform action; this requires reflective evaluation as KTA activities are undertaken and efforts are focussed to revise actions. Such feedback loops contribute to generating pertinent research evidence that is ready for use by policy and practice organizations. For example, INSPIRE created a virtual environment where diverse stakeholders can access and integrate data and information from their different sources to support continuous quality improvement in the delivery of empirically supported interventions. INSPIRE also facilitated gathering of standardized data from many institutions, thus creating a rich data source for studying implementation and outcomes across organizations.

\section{4) Systems interventions are not projects}

Some cases faced pressure to produce tangible, short-term outputs. Focussing on systems-level processes and encouraging other organizations to fill identified gaps means that the collaborative efforts are directed at finding new actors, igniting interest in a high-level agenda, and encouraging other organizations with longer-term mandates and with relevant expertise to take leadership on specific actions. For example, SiG Causeway was careful not to create a cumbersome governance structure with ambitions to develop and implement a series of projects. Instead they created a core network of highly engaged leaders drawn from divergent fields, all guided by an overarching strategic framework aimed at igniting cross-sector leadership and shared learning experiences to advance social finance in Canada. These leaders convened showcase and learning events across Canada, hosted webinars to link organizations across sectors to discuss social finance and helped create and populate a common web hub / portal with guides, resources and white papers for translating the concepts into action.

\section{5) Different kinds of supports are needed at different times in different contexts}

Even though objectives stayed the same within cases over time, their KTA activities and strategies changed frequently, adapting to the needs of their partners and clients. Cases drew from a diverse range of KTA options and selected those that were the best fit for the time, place, people and purpose. For example, ResearchImpact customized each KTA initiative from a suite of activities that they developed over time. Similarly, the United Way Community of Practice had a range of events for which people can self-select depending on their interests.

\section{Discussion}

The 5 guidelines on practical strategies to implement systems approaches to KTA reinforce a subset of system principles. The importance of relationships was particularly reinforced, especially enduring relationships between individuals and groups with diverse perspectives, including from research, policy and practice. Also reinforced was the importance of adapting principles to diverse and dynamic contexts, and feedback for continuous learning. Consistent with the goal of the scan, we identified practical examples of applying these principles. Nonetheless, the results represent a modest start to providing useful insights and guidance to better translate system concepts for KTA into practice. Reflecting on the results, the advisory group identified 3 promising directions for KTA to solve complex problems.

\section{1) Embrace emergent and holistic approaches}

Systems approaches challenge assumptions about expectations, guarantees, final answers, and "control," especially by scientists and specialists with particular expertise. In complex and dynamic systems, no patterns stay in place for long and results of interventions may not have the certainty science usually views as desirable. Therefore we need to avoid formulaic approaches, especially across varied contexts; for example, it is not appropriate to replicate a successful intervention with fidelity across diverse communities and 
populations. However, recognizing the importance of context does not prevent identifying core components for success and gleaning meaning across diverse contexts or documenting emergent conditions as was done with Tamarack's Vibrant Communities and SiG Causeway work. The development and use of context-sensitive research and evaluation methods are required to learn about what works and how and why. These insights will inform appropriate adaptations of interventions.

Emergent and holistic approaches also require continuous learning. Efforts are needed to continue to evaluate and learn from adaptations and study the processes of refining and scaling up interventions. ${ }^{37}$ This creates opportunities to further develop novel and participatory models for conducting evaluations that focus on learning within dynamic environments - in contrast to results-based evaluations that may focus on accountability with little to no consideration of context.

\section{2) Focus on enduring relationships that are solution-oriented}

To have an effect, enduring relationships are needed between the policy, practice and research sectors that can nimbly identify, test and adapt solutions to complex problems. During times of limited resources, relationship-building activities can easily be considered luxuries that do not warrant time or funding. However, inclusion of developmental periods for project funding may be an essential ingredient to building effective and relevant interventions and appropriate research methods to harness and use evidence to effect change.

The scan findings from United Way Toronto and the National Treatment Strategy indicate that investment in and the use of community of practice models, though varied in their structure, purpose and aims, may also hold promise in this area. Community of practice models, guided by systems principles, would focus on contextsensitive questions, be oriented toward ongoing learning and diverse exchange, emphasize emergence, value different perspectives and sources of knowledge, and aim to understand the implementation context. Seeking diversity in collaborators and perspectives to engage in solving complex problems implies inclusivity; a conscious bringing together of people who do not necessarily share perspectives, culture or language to work across differences. Power relationships need to be acknowledged and addressed within these collaborations (e.g. how do we give equal voice to participants of different race, gender, class, education). The time and effort required to build enduring and diverse relationships are worthwhile, especially to make sure they are created authentically and sustained.

\section{3) Address gaps in the system in addition to gaps in the evidence}

Knowledge development in public health normally focusses on filling gaps in evidence, and especially on providing detailed descriptions of problems. Attention to studying policy and program interventions has been growing recently. ${ }^{11,38}$ Consistent with models of places to intervene in complex systems, ${ }^{3,4}$ the scan results point to the importance of structural changes (e.g. multistakeholder teams, networks) and feedback as valuable system interventions. A considerable challenge is limited funding to address these structural elements and other system gaps such as feedback through common measurement approaches across organizations and jurisdictions. ${ }^{30}$

\section{Strengths and limitations}

The main strengths of this scan are the importance and relevance of its goals for advancing effective KTA on complex issues such as chronic disease prevention, and the methods we used.

The public health field has placed much more emphasis on defining problems than on developing solutions. Intervening through policies, programs and system changes to address the urgent, serious and complex problems in public health, especially chronic diseases, means working in new ways, including new ways to create and apply knowledge that respects the complexity of the problems. This scan contributes to figuring out how promising approaches to KTA can be implemented in practice.

The strength of our results is enhanced by the methods we used to identify and select a broad range of cases for the scan. The sampling methods resulted in a sizable and manageable number of relevant cases with substantial diversity. Results were also strengthened by the systematic approach to the selection, collection and analysis of information within and across cases and the use of information from both documents and interviews.

The scan was limited by its modest scope because of limited resources. The scan was designed as a preliminary and exploratory analysis. Results suggest there is much to learn about KTA for solving complex problems from existing efforts. More in-depth qualitative and quantitative study and observation of KTA initiatives using system approaches would yield more extensive insights. Learning over a longer period of time from the cases included in the scan and others would also be useful, especially to better understand ways to facilitate dynamic KTA processes and their effectiveness under different conditions.

\section{Conclusion}

Ways to address the burden of chronic disease must respect the complexity of these problems and related environments, including the nature of the knowledge needed to address such problems and how and why knowledge is created and used. Learning from existing examples of systems approaches to KTA can identify practical implementation strategies. These strategies may be enhanced by emphasizing a holistic science with a focus on adaptation of interventions, strengthening enduring relationships that bring together diverse perspectives and assets, and addressing gaps in the system in addition to gaps in the evidence. In turn, at least small gains in changing environments, organizations and behaviours to solve complex public health problems can be achieved.

\section{Acknowledgements}

This project was funded by the Public Health Agency of Canada. Contributions from Barbara Riley were supported by a grant from the Canadian Cancer Society (grant \# 2011-701019). 


\section{References}

1. Rittel HW, Weber MM. Dilemmas in a general theory of planning. Policy Sci. 1973;4(2):155-69.

2. Kreuter MW, De Rosa C, Howze EH, Baldwin GT. Understanding wicked problems: a key to advancing environmental health promotion. Health Educ Behav. 2004;31(4):441-54.

3. Finegood DT. The complex systems science of obesity. In: Cawley J, editor. The Oxford handbook of the social science of obesity. Oxford (UK): Oxford University Press; 2011; p. 208-36.

4. Meadows DH. Thinking in systems: a primer. Wright D, editor. White River Junction (VT): Chelsea Green Publishing; 2008. $240 \mathrm{p}$.

5. Green LW, Glasgow RE, Atkins D, Stange K. Making evidence from research more relevant, useful, and actionable in policy, program planning, and practice: slips "Twixt cup and lip". Am J Prev Med. 2009;37(6 Suppl 1):S187-91.

6. Frenck J. Balancing relevance and excellence: organizational responses to link research with decision-making. Soc Sci Med. 1992; 35:1397-404.

7. Best A, Terpstra JL, Moor G, Riley B, Norman CD, Glasgow RE. Building knowledge integration systems for evidenceinformed decisions. J Health Organ Manag. 2009;23(6):627-41.

8. Best A, Holmes B. Systems thinking, knowledge and action: towards better models and methods. Evid Policy. 2010;6(2):145-59.

9. Best A, Trochim W, Haggerty J, Moor G, Norman CD. Systems thinking for knowledge integration: new models for policyresearch collaboration. In: Organizing and reorganizing: power and change in health care organizations. McKee L, Ferlie E, Hyde P, editors. London (UK): Routledge; 2008: p. 154-66.
10. Holmes BJ, Finegood DT, Riley BL, Best A. Systems thinking in dissemination and implementation research. In: Dissemination and implementation research in health: translating science to practice. Brownson RC, Colditz GA, Proctor EK, editors. New York (US): Oxford University Press; 2012. p. 175-91.

11. Cameron R, Riley BL, Campbell HS, Manske S, Lamers-Bellio K. The imperative of strategic alignment across organizations: the experience of the Canadian Cancer Society's Centre for Behavioural Research and Program Evaluation. Can J Public Health. 2009;100(1):Suppl I27-30.

12. Global status report on noncommunicable diseases, 2010. Geneva (CH): World Health Organization; 2011. 176 p.

13. Canadian Cancer Statistics 2007. Toronto (ON): Canadian Cancer Society/ National Cancer Institute of Canada; 2007. 114 p.

14. Department of chronic diseases and health promotion. Preventing chronic diseases: a vital investment [Internet]. Geneva $(\mathrm{CH})$ : World Health Organization; 2005 [cited 2014 May 21]. 200 p. Available from: http://www.who.int/chp/chronic_disease_ report/en/index.html

15. Hu FB, Manson JE, Stampfer MJ, et al. Diet, lifestyle, and the risk of type 2 diabetes mellitus in women. N Engl J Med. 2001; 345(11):790-7.

16. Dobrow MJ, Goel V, Upshur RE. Evidencebased health policy: context and utilisation. Soc Sci Med. 2004;58(1):207-17.

17. Rose G. The strategy of preventive medicine. Oxford (UK): Oxford University Press; 1993. $135 \mathrm{p}$.

18. Vandenbroek P, Goossens J, Clemens M. Tackling obesities: future choices - building the obesity system map [Internet]. (UK): Government Office for Science; 2007 [cited 2014 May 21]. Available from: https://www .gov.uk/government/publications/reducing -obesity-obesity-system-map

19. Lavis JN, Davies HTO, Gruen RL, Walshe $\mathrm{K}$, Farquhar CM. Working within and beyond the Cochrane collaboration to make systematic reviews more useful to healthcare managers and policy makers. Healthc Policy. 2006;1(2):21-33.
20. Brownson RC, Fielding JE, Maylah CM. Evidence-based public health: a fundamental concept for public health practice. Ann Rev Public Health. 2009; Jan 14; 30: 175201.

21. Kiefer L, Frank J, Di Ruggiero E, et al. Fostering evidence-based decision-making in Canada: examining the need for a Canadian population and public health evidence centre and research network. Can J Public Health. 2005;96(3):111-9.

22. Riley B, Edwards N. A primer on multiple intervention programs and some implications for a research agenda [Internet]. Ottawa (ON): CHRU \& NBPRU; 2009 [cited 2014 May 21]. 22 p. Publication No. M20091. Available from: http://aix1.uottawa.ca / nedwards/chru/english/pdf/M2009-1.pdf

23. Hobin EP, Hayward S, Riley B, Di Ruggiero E, Birdsell J. Maximising the use of evidence: exploring the intersection between population health intervention research and knowledge translation from a Canadian perspective. Evid Policy. 2012;8(1):97-115

24. Green LW. From research to "best practices" in other settings and populations. Am J Health Behav. 2001;25(3):165-78.

25. Graham ID, Logan J, Harrison MB, et al. Lost in knowledge translation: time for a map. J Contin Educ Health Prof. 2006;26(1):13-24.

26. Best A, Clark P, Leischow S, Trochim W, editors. Transforming tobacco control through systems thinking: integrating research and practice to improve outcomes. Smoking and Tobacco Control Monograph 18. Bethesda (MD): Department of Health and Human Services, Public Health Service, National Institutes of Health, National Cancer Institute, NIH: 2007. 320 p

27. Finegood DT, Johnston L, Giabbanelli P, et al. Complexity and systems thinking. In LW. Green, editor [Internet]. New York: Oxford Bibliographies Online, Public Health; 2012. [cited 2014 May 21]. Available from: http:// www.oxfordbibliographies.com/view/document /obo-9780199756797/obo-9780199756797-0049 .xml? rskey $=$ VJCfVs\&result $=11 \& q=$

28. Riley B, Norman C, Best A. Knowledge integration in public health: a rapid review using systems thinking. Evid Policy. 2012 Nov; 8(4):417-32. 
29. Institute of Medicine. Bridging the evidence gap in obesity prevention: a framework to inform decision making. Kumaniyika SK, Parker L, Sim LJ, editors. Washington (DC): The National Academies Press; 2010. 336 p.

30. Institute of Medicine. Evaluating obesity prevention efforts: a plan for measuring progress. Green LW, Sim L, Breiner H, editors. Washington (DC): The National Academies Press; 2013. 462 p.

31. Foster-Fishman PG, Nowell B, Yang H. Putting the system back into systems change: a framework for understanding and changing organizational and community systems. Am J Community Psychol. 2007;39(3/4): 197-216

32. Leischow SJ, Best A, Trochim WM, et al. Systems thinking to improve the public's health. Am J Prev Med. 2008;35(2S): S196203.

33. Gamble J, Penney T. Systems approaches to knowledge mobilization: scan of initiatives. Ottawa (ON): Chronic Disease Interventions Division, Public Health Agency of Canada; 2012.

34. Bar-Yam Y. Complex systems and sports: complex systems insights to building effective teams [Internet]. Cambridge (MA): NECSI; 2003 [cited 2014 May 21]. 8 p. Available from: http://necsi.edu/projects /yaneer/SportsBarYam.pdf

35. Bar-Yam Y. Making things work: solving complex problems in a complex world. Cambridge (MA): NECSI Knowledge Press; 2005. 306 p.

36. Wheatley M, Frieze D. Using emergence to take social innovation to scale [Internet]. Spokane (WA): The Berkana Institute; 2006 [cited 2014 May 21]. 7p. Available from: http:// margaretwheatley.com/articles/emergence .html

37. Patton MQ. Developmental evaluation: applying complexity concepts to enhance innovation and use. 1st ed. New York: The Guilford Press. 2010 Jun 15. 375 p.

38. Hawe P, Potvin L. What is population health intervention research? Can J Public Health. 2009;100(Suppl 1):I8-14. 